\title{
Lung Ultrasound for the Detection of Pneumothorax Might Be Misleading in Patients Following Lung Transplantation
}

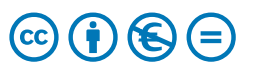

\author{
Authors \\ Ines Schroeder, Thomas Weig, Lorenz Frey, Patrick Scheiermann \\ Affiliation \\ Department of Anesthesiology, University Hospital Ludwig- \\ Maximilians-University Munich, Munich, Germany \\ Key words \\ ultrasound, pneumothorax, lung transplantation \\ received 07.02 .2017 \\ revised $\quad 07.02 .2017$ \\ accepted 02.04.2017
}

\author{
Bibliography \\ DOI https://doi.org/10.1055/s-0043-108992 \\ Ultrasound Int Open 2017; 3: E128-E129 \\ (c) Georg Thieme Verlag KG Stuttgart · New York \\ ISSN 2199-7152 \\ Correspondence \\ PD Dr. Dr. Patrick Scheiermann \\ University Hospital Ludwig-Maximilians-University Munich \\ Anesthesiology \\ Marchioninistr. 15 \\ 81377, Munich \\ Germany \\ Tel.: + 49/89/4400 73410 \\ patrick.scheiermann@med.uni-muenchen.de
}

Dear Editor,

Lung ultrasound (LUS) for the detection of pneumothorax (PTX) is widely accepted in critical care medicine. The sensitivity of LUS in the detection of PTX is higher than that of conventional anterior-posterior chest radiography and similar to that of computerized tomography (CT) [1]. Recommendations for LUS suggest a stepby-step approach to detect important findings that are relevant for the diagnosis of PTX: the absence of lung sliding, B-lines, and lung pulse as well as the presence of the lung point. In an unstable patient, the absence of either horizontal (lung sliding) or vertical (lung pulse) movement of the pleural line combined with the absence of B-lines allows prompt and safe diagnosis of PTX without the need for searching the lung point [2].

Patients after lung transplantation (LuTx) are at high risk for developing PTX. Occult PTX in supine chest radiography is a wellknown problem. In addition, every transfer to the CT suite bears a considerable risk for patients following LuTx, since these patients are often hemodynamically instable. Consequently, bedside LUS seems to be an ideal tool to detect PTX in this situation.

Doing routine LUS in patients following LuTX, we noticed that it presented differently from what we had expected. We describe one patient, whose LUS did not show any horizontal or vertical movement of the pleural line (i. e., absence of lung sliding and B-lines on B-mode (not shown) as well as absence of the sea shore sign and lung pulse on M-mode ( $\triangleright$ Fig. 1a) on the right side of the chest, thus suggesting unilateral PTX according to the guidelines [2]. On the left hemithorax, the sonographic detection of lung pulse ruled out major PTX ( $\triangleright$ Fig. 1b). A lung point could not be detected anywhere. The same day CT scan ruled out PTX on the right hemithorax. However, it did reveal a small anterior PTX on the left hemithorax ( $\triangleright$ Fig. 1c). The patient had undergone bilateral LuTx 12 weeks earlier due to pulmonary hypertension with a complicated postoperative course due to infection, pleural empyema and weaning failure due to critical illness polyneuropathy and was still being treated in the intensive care unit (ICU). Written informed consent was obtained from the patient prior to submission of this manuscript.

The absence of lung sliding without PTX has been observed in the case of pleural adhesions, which may mimic a motionless pleural line [1]. Isolated B-lines can be found in healthy patients but the absence of B-lines is considered without pathology [3]. In our opinion, LUS is still a valuable tool in the hands of experienced investigators. Nevertheless, it should be used with caution in LuTx recipients in the ICU setting as bedside LUS for the detection of PTX might be misleading in these patients due to altered movement of the pleural line. As far as we know, there has not been any systematic approach with LUS in patients following LuTx. According to the findings in this report, a systematic trial to evaluate LUS in patients after LuTx is needed. 

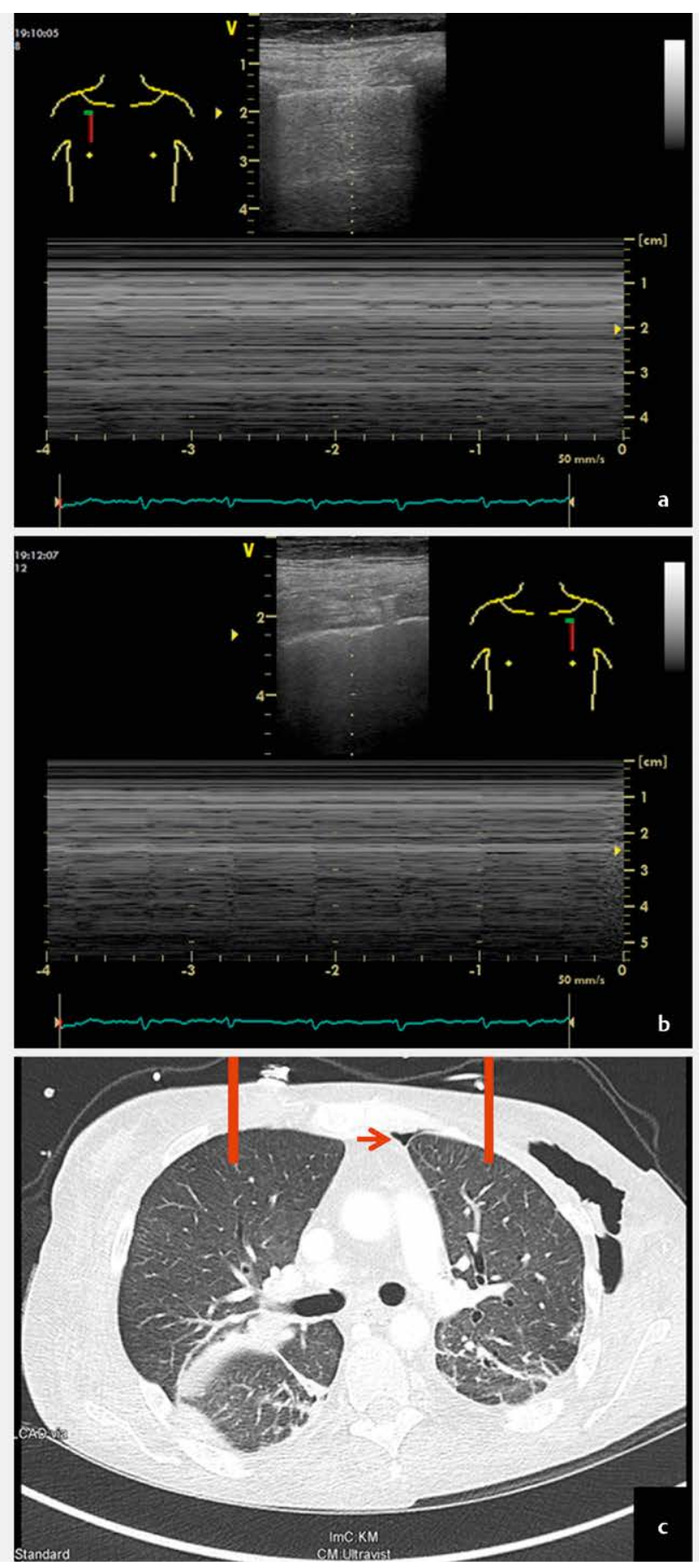

- Fig. 1 Lung ultrasound findings and chest computed tomography (CT) in a patient 12 weeks after bilateral lung transplantation. a $\mathrm{M}$-mode of the right hemithorax shows the absence of the sea shore sign and the absence of the lung pulse. The position of the linear transducer (General Electric, Vivid S6, 12L) is indicated with a body mark. Simultaneous electrocardiographic (ECG) recordings are shown. $\mathbf{b}$ M-mode of the left hemithorax shows the absence of the sea shore sign but the presence of the lung pulse. The position of the linear transducer (General Electric, Vivid S6, 12L) is indicated with a body mark. Simultaneous ECG recordings are shown. c Chest CT of the same patient performed on the same day as the ultrasound examination ruling out major bilateral pneumothorax (PTX). The approximate positions of the linear transducers for the M-mode images as obtained in $\mathbf{a}$ and $\mathbf{b}$ are indicted as red bars. Note the small anterior PTX on the left hemithorax (red arrow).

\section{References}

[1] Volpicelli G. Sonographic diagnosis of pneumothorax. Intensive Care Med 2011; 37: 224-232

[2] Volpicelli G, Elbarbary M, Blaivas M et al. International evidence-based recommendations for point-of-care lung ultrasound. Intensive Care Med 2012; 38: 577-591

[3] Ciccarese F, Chiesa AM, Feletti F et al. The senile lung as a possible source of pitfalls on chest ultrasonography and computed tomography. Respiration 2015; 90: 56-62 